women receiving physical examinations for any HPV subtypes, HR HPV subtypes, 9-valent HPV and 4-valent HPV.

Conclusion Our study delineated the distribution and trend of type-specific HPV among both gynecological outpatients and women receiving physical examinations in Guangdong, which may provide valuable data to inform cervical cancer screening and HPV vaccination programs for women in this province.

Disclosure No significant relationships.

\section{P835 QUANTITATIVE ORAL HPV16 AND HPV18 DETECTION IN PATIENTS ATTENDING DENTAL CLINICS}

${ }^{1}$ Helen Stankiewicz Karita*, ${ }^{2}$ Amalia Magaret, ${ }^{3}$ Quinne Feng, ${ }^{4}$ Anna Wald. ${ }^{1}$ University of Washington, Seattle, USA; ${ }^{2}$ University of Washington; Fred Hutchinson Cancer Research Center, Department of Biostatistics and Department of Laboratory Medicine, University of Washington; Department of Public Health Science, Fred Hutchinson Cancer Research Center, Seattle, USA; ${ }^{3}$ Fidalab, Seattle, USA; ${ }^{4}$ University of Washington; Fred Hutchinson Cancer Research Center, Department of Medicine, Department of Laboratory Medicine, Department of Epidemiology, University of Washington; Vaccine and Infectious Diseases Division, Fred Hutchinson Cancer Research Center, Seattle, USA

10.1136/sextrans-2019-sti.880

Background The incidence of HPV-associated oropharyngeal cancer is increasing substantially, especially among men. Our goal was to assess quantitative HPV16 and HPV18 detection in oral rinses obtained in dental offices in Seattle, Washington. Methods We evaluated 15,313 oral rinses collected for during routine dental visits from 11/2016 to 11/2018. Multiplex Taqman qPCR was utilized to determine HPV16 and HPV18 viral load (VL).

Results In persons with a single sample, oral HPV was detected in $152(1 \%)$ persons: $127(0.83 \%)$ were HPV16 positive and $25(0.16 \%)$ were HPV18 positive. HPV16 was detected in $1.4 \%$ of men; the median age was 55 and median VL was 39.7 (range 0.1 - 589855.2 copies $/ \mathrm{mL}$ ). Only $0.4 \%$ of samples were HPV16 positive in women (median age 48, median VL 1.08, range 0.01 - 825 copies $/ \mathrm{mL})$. HPV18 was detected in $13(0.18 \%)$ men and $12(0.14 \%)$ women. A second oral rinse was collected in 628 persons (mean 6 months apart): 581 were HPV negative at baseline and only one became HPV16 positive at second rinse, 39 were HPV16 positive at baseline and 13 remained HPV16 positive at the second rinse, and 8 were HPV18 positive at baseline and 2 remained HPV18 positive at subsequent rinse. Patients with consecutive positive tests were all men and had higher baseline median VL (385 vs 0.90 HPV16 copies/mL; 24 vs 0.80 HPV18 copies $/ \mathrm{mL}$ ) compared to those with first positive and second negative samples.

Conclusion Oral rinse is an acceptable method of HPV testing and patients seen for routine dental care are interested in testing. Comparable to published studies, oral HPV was more frequent among men than women, especially at higher VL levels. HPV16 persistence was more common in those with high VL at baseline test. Future studies are needed to evaluate the feasibility of an effective primary and secondary screening strategy for oropharyngeal cancer using quantitative oral HPV detection.

Disclosure No significant relationships.
P836

TRENDS IN ANOGENITAL WARTS SINCE INTRODUCTION OF HUMAN PAPILLOMAVIRUS VACCINES IN CONNECTICUT, USA

${ }^{1}$ Linda Niccolai*, ${ }^{2}$ Monica Brackney. ${ }^{1}$ Yale School of Public Health, Epidemiology of Microbial Diseases, New Haven, USA; ${ }^{2}$ Yale School of Public Health, CT Emerging Infections Program, New Haven, USA

\subsection{6/sextrans-2019-sti.88}

Background HPV vaccines have been available in the US since 2006 and have the potential to prevent $\geq 90 \%$ of anogenital warts (AGW). Monitoring trends in AGW is important to assess progress of immunization programs.

Methods Two datasets for Connecticut (population 3.6 million) were used. Data for residents with private insurance were available for 2012-2017 from a multi-payer claims database $(\sim 1$ million covered individuals per year). Data for residents with Medicaid insurance were available for 2009-2013 $(\sim 512,000$ individuals per year).

Results Among privately insured women, the annual incidence rate (IR) of AGW declined from 104 to 68 per 100,000 during 2012-2017. Significant declines were observed for women aged 15-19 ( $\mathrm{p}$ for trend $=.03$, average annual percent change (AAPC) -11\%), 20-24 (p<0.001, AAPC -13\%), 25-29 $(\mathrm{p}<0.001$, AAPC $-7 \%)$, and 30-34 $(\mathrm{p}<0.01$, AAPC $-2 \%)$. Similar patterns were observed among men, with an overall decline from 179 to 105 per 100,000 , and $p<0.001$ for trends in each age group and AAPCs ranging from $-16 \%$ to 3\%. Among Medicaid-insured women, the overall IR of AGW declined from 175 to 145 per 100,000 during 2009-2013. Significant declines were observed for women up to age 29 years $(\mathrm{p}<0.05$ and AAPCs from $-13 \%$ to $-3 \%$ ). Rates in Medicaid-covered men did not decline in any age group.

Conclusion In Connecticut, significant and substantial declines in AGW have occurred in women during both periods (20092013 and 2012-2017). In men, declines occurred during 2012-2017 but not during 2009-2013, perhaps due in part to the later routine recommendation for males in $2011 \mathrm{com}$ pared to females in 2006. Greater declines in younger populations are consistent with HPV vaccine impact. These reductions have been achieved in a setting of moderate HPV vaccine uptake and could be further reduced with higher coverage.

Disclosure No significant relationships.

\section{P837 HPV16/18 VACCINE: INFLUENCE ON THE SYSTEMIC AND LOCAL TH1/TH2 CYTOKINE PROFILE}

${ }^{1}$ Paulo Giraldo, ${ }^{2}$ José Sanches, ${ }^{1}$ Rose Luce Do Amaral ${ }^{*},{ }^{1}$ Isabel Migliorini De Oliveira, ${ }^{2}$ Cristiane Gil, ${ }^{1}$ Michelle Discacciati. ${ }^{1}$ UNICAMP, Faculdade De Ciências Médicas, Campinas, Brazil; ${ }^{2}$ UNIFESP, Departamento de Morfologia e Genética, São Paulo, Brazil

\subsection{6/sextrans-2019-sti.882}

Background The immunological mechanism of the vaccines acts systemically in order to prevent a specifically infection. Although HPV-antibodies levels have been studied in serum and cervicovaginal (CVC) samples of vaccinated women, the TH1/TH2 cytokines levels has not yet been adequately characterized. In our study, we investigated the effects of the 
Human Papillomavirus (HPV-16/18) AS04-adjuvanted vaccine (Cervarix ${ }^{\circledR}$ ) on the serum and cervicovaginal microenvironment, characterizing the $\mathrm{TH} 1 / \mathrm{TH} 2$ cytokines profile.

Methods A subset of 20 women between 18 and 40 years old without genital coinfections (bacterial vaginosis, Herpes virus, Candida sp Neisseira gonorrhea or Chlamydia trachomatis) were selected to receive the three doses of the HPV-16/18 AS04-adjuvanted vaccine $\left(\right.$ Cervarix ${ }^{\circledR}$ ). Blood and cervicovaginal samples were collected before the first dose and 30 days after the third dose. TH1 (INF- $\gamma$, IL2, IL-12p70, TNF- $\alpha$, GM-CSF) and TH2 (IL-4, IL-5, IL-10, IL-13) cytokines were determined by Immunology Multiplex.

Results In the blood samples there were no statistically significant differences in the level of cytokines before or after the three doses of the vaccine, except for TNF- $\alpha$ (INF- $\gamma$ : $\mathrm{p}=0.797 ; \quad$ IL2: $\mathrm{p}=0.735, \quad$ IL-12p70: $\mathrm{p}=0.881 ;$ TNF- $\alpha$ : $\mathrm{p}=0.011$, GM-CSF, $\mathrm{p}=0.721 ; \mathrm{IL}-4, \mathrm{p}=0.223 ; \mathrm{IL}-5, \mathrm{p}=0.860$; IL-10, $p=0.473$; IL-13, $p=0,913$.). However, for the CVC samples, there was a tendency to decrease the cytokine level after the three doses of the vaccine. This decrease was significant for the INF- $\gamma(p=0,010), \quad$ IL-5, $(p=0,005)$, IL-12p70 $(\mathrm{p}=0,002)$ e IL-13 ( $\mathrm{p}=0,002)$.

Conclusion TH1/TH2 cytokines were detected in serum of women who received the HPV-16/18 AS04-adjuvanted vaccine, but there were no significant differences before and after the three doses. In the vaginal samples there was a significant decrease of INF- $\gamma$, IL-12p70, IL-5 and IL-13. Understanding the clinical significance of these modifications is a very relevant issue and future studies that address the network of inflammatory and anti-inflammatory cytokine effects should be considered.

Disclosure No significant relationships.

\section{P838 ASSOCIATIONS OF THE VAGINAL MICROBIOTA WITH HPV INFECTION AND CERVICAL DYSPLASIA IN SOUTH AFRICAN WOMEN LIVING WITH HIV}

${ }^{1} J a n n e k e$ Van De Wijgert*, 'Allessandra Gill, ${ }^{1}$ Alistair Darby, ${ }^{2}$ Helen Kelly, ${ }^{3}$ Admire Chikandiwa, ${ }^{4}$ Sinead Delany-Moretlwe, ${ }^{5}$ Marijn Verwijs, ${ }^{6}$ Suzanna Francis, ${ }^{6}$ Philippe Mayaud. 'University of Liverpool, Liverpool, UK; ${ }^{2}$ London School of Hygiene and Tropical Medicine, London, UK; ${ }^{3}$ Wits RHI, University of the Witwatersrand, Johannesburg, South Africa; ${ }^{4}$ University of the Witwatersrand, Wits RHI, Johannesburg, South Africa; ${ }^{5}$ University of Liverpool, Institute of Infection and Global Health, Liverpool, UK; ${ }^{6}$ London School of Hygiene and Tropical Medicine, London, UK

\subsection{6/sextrans-2019-sti.883}

Background Fifteen longitudinal studies have shown associations between bacterial vaginosis and high risk human papillomavirus (hrHPV) acquisition and/or persistence, and/or cervical dysplasia. However, few studies assessed the vaginal microbiota (VMB) comprehensively, and none controlled the dysplasia association for persistent hrHPV.

Methods 623 women attending HIV outpatient clinics in Johannesburg, South Africa, were examined for hrHPV (InnoLipA HPV Genotyping Extra Assay), cervical dysplasia (histology), and vaginal microbiota (VMB; V3-V4 Illumina HiSeq 2x300bp with Swarm OTU-picking) at baseline and endline, a median of 16 months after baseline. VMB research questions were addressed in two nested case-control designs.

Results Hierarchical clustering resulted in seven VMB types: $L$. iners-dominated ( $\mathrm{Li} ; \mathrm{n}=214$ samples), Lactobacillus crispatus or L. jensenii-dominated (Lcj; $\mathrm{n}=68)$, Bifidobacterium-dominated (BD; $\mathrm{n}=2)$, lactobacilli + bacterial vaginosis (BV)- anaerobes $(\mathrm{L}+\mathrm{A} ; \mathrm{n}=208)$, BV-like (BV; $\mathrm{n}=303)$; BV-anaerobe dominated $(\mathrm{AD} ; \mathrm{n}=56)$; and pathobiont-characterised $(\mathrm{PB}$; $\mathrm{n}=19$ ). Women with new or persistent hrHPV during followup were less likely to have an Lcj VMB type (compared to Li) at endline, and persistent hrHPV was associated with vaginal anaerobic dysbiosis at baseline (decreased lactobacilli, increased BV-anaerobes, and increased Nugent score). Women who developed CIN2+, compared to women with persistent hrHPV but no CIN2+, were more likely to have vaginal anaerobic dysbiosis at endline (decreased lactobacilli, increased BV-anaerobes, and increased diversity), but not at baseline. These associations persisted after controlling for age, hormonal contraception, and CD4+ count; several additional potential confounders (HIV plasma viral load, antiretroviral therapy, sexually transmitted infections, sexual risk-taking, among others) were evaluated.

Conclusion Frequent hrHPV exposure (and/or increased sexual risk-taking) likely causes vaginal dysbiosis, but a bilateral relationship cannot be ruled out. Women with vaginal dysbiosis are not at increased risk of CIN2+ development when hrHPV status is taken into account, but vaginal dysbiosis does develop when CIN2+ lesions develop. These results should be confirmed in even larger longitudinal studies.

Disclosure No significant relationships.

\section{P839 A CHEMICALLY MODIFIED $\beta$-LACTOGLOBULIN (JB01) IS EFFECTIVE IN TREATING HPV INFECTION AND PREVENTING SEXUAL TRANSMISSION OF HIV}

Shibo Jiang*. Fudan University, School of Basic Medical Sciences, Shanghai, China

\subsection{6/sextrans-2019-sti.884}

Background More than $90 \%$ of the new HIV infection in China occurred through sexual transmission, particularly among the men who have sex with men (MSM). Cervical cancer, the second most common cancer among women, is caused by sexually acquired infection with high-risk types of HPV, such as types 16 and 18 . We previously have shown that a chemically modified bovine milk protein, $\beta$-lactoglobulin (3HP$\beta$-LG, also known as JB01), is effective against infection by a broad-spectrum of HIV (Nat. Med., 2:230,1996) and HPV entry inhibitor. Therefore, we intended to develop topical formulations containing JB01 against HIV and HPV infections.

Methods A pseudotyped HPV particles expressing HPV L1 and L2 proteins were used for testing the inhibitory activity of JB01. A randomized open-label clinical trial of a JB01 biological dressing (JB01-BD) administered intravaginally was performed to evaluate its in vivo safety and efficacy. Both pseudotyped and live HIV-1 strains with different subtypes and tropisms were used for evaluating the in vitro efficacy of JB01 and a non-human primate (NHP) model was used for testing the in vivo efficacy of JB01.

Results The trial of JB01-BD administered intravaginally demonstrated that JB01-BD is safe and effective. About $60.5 \%$ and $13.5 \% \mathrm{HPV}$-positive women in the treatment and nontreatment groups, respectively, became HPV-negative $(P<$ 0.001). In vitro study suggests that JB01 exhibits broad-spectrum antiviral activity against divergent HIV-1 strains, including those resistant to the current antiretroviral therapeutics. Rhesus macaques monkeys pretreated with a topical formulation were protected against rectal challenge with SHIVSF162P3. 